\title{
Luminescence from laser-created bubbles in cryogenic liquids
}

\author{
Ohan Baghdassarian, Bernd Tabbert, Gary A. Williams ${ }^{1}$ \\ Department of Physics and Astronomy, University of California, Los Angeles, CA 90095, USA
}

\begin{abstract}
A luminescence pulse has been observed from a laser-created bubble in liquid nitrogen and liquid argon at the first collapse point of the bubble. An unusual feature is that the width of the pulse is of order 100-1000 ns, much longer than the 2-8 ns pulses observed when the same experiment is carried out with a water sample.
\end{abstract}

Keywords:

Bubble luminescence; Liquid nitrogen; Liquid argon; Sonoluminescence; Bubble dynamics;

Light emission from bubbles acoustically trapped in water has been intensively studied over the last few years [1], known as single-bubble sonoluminescence (SBSL). Since the early 70's there have been sketchy reports in the literature that luminescence could also be observed from bubbles in liquid nitrogen $[2,3]$. However, initial attempts to observe SBSL in liquid nitrogen in our lab were unable to observe any light emission from trapped helium bubbles [4]. In this paper a different technique to generate luminescing bubbles in liquid nitrogen and liquid argon is described and compared to similar measurements in water $[5,6]$.

The bubbles are created by a Nd:YAG laser in a stainless steel sample cell mounted inside an optical cryostat, which can be pressurized up to 35 bar at temperatures between 65 and $90 \mathrm{~K}$. Ultrapure argon or nitrogen $(5 \mathrm{~N})$ is condensed into the cell. The light emitted by an argon-ion laser passes through two sets of quartz windows and backlights

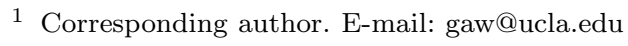

the focus region of the YAG pulse. The attenuation of the beam by a created bubble is monitored by a photodiode, yielding the time dependence of the bubble radius. A photomultiplier is also used to monitor any light emission from the bubble. For imaging purposes the photodiode is replaced by a CCD camera attached to a long-distance microscope, and the laser is pulsed using an acoustooptic modulator. By these means pictures of the bubble can be taken with exposure times as short as $500 \mathrm{~ns}$.

Fig. 1 shows the dynamics and luminescence of a laser-created bubble in liquid nitrogen. The YAG pulse at $\mathrm{t}=0$ ionizes the liquid at the focal point and there is a flash from the recombining plasma. The bubble expands to a maximum radius of about $1 \mathrm{~mm}$, while a decaying background luminescence is observed, probably from recombining metastables such as $\mathrm{N}_{2} \mathrm{~A}^{3} \Sigma_{u}^{+}$excited molecules produced in the plasma flash. Exactly coincident with the bubble collapse point at $52 \mu \mathrm{s}$, a sharp lumines- 


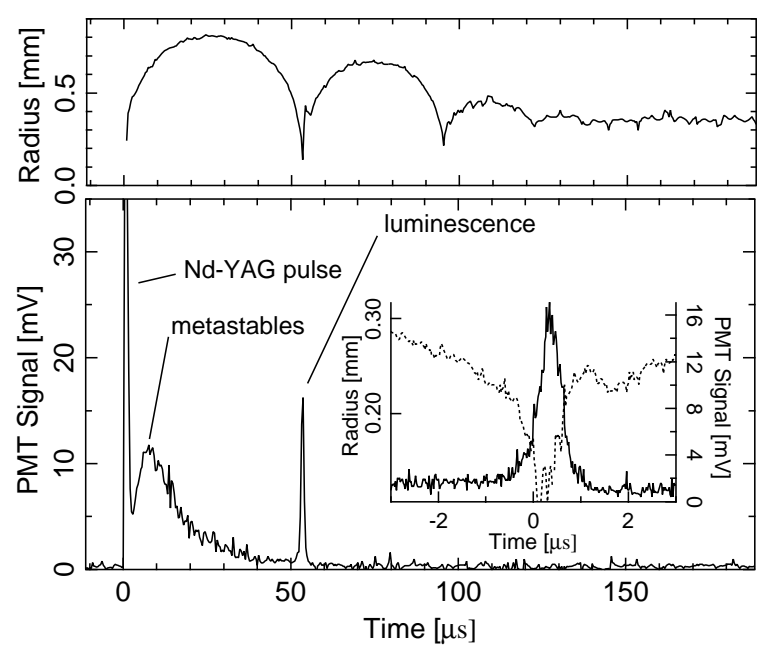

Fig. 1. Dynamics and light emission of a laser-created bubble in liquid $\mathrm{N}_{2}$ at $66 \mathrm{~K}$ and 5.8 bar; upper trace: radius-time curve showing large bounces; lower trace: PMT signal showing the plasma emission at $\mathrm{t}=0$, the recombination of metastable species and the luminescence peak; inset: expanded view of a similar luminescence pulse (solid line) and radius-time curve (dashed line).

cence pulse is observed on the PMT. After the luminescence pulse the bubble rebounds with an afterbounce that is unusually large compared to those in water $[1,5]$. This behavior appears to be similar to the very large afterbounces observed in acoustically driven helium bubbles in liquid nitrogen [4].

The inset of Fig. 1 shows one of the luminescent pulses on an expanded time scale; the unusual feature of these pulses compared to those observed from laser-created bubbles in water [5] is their relatively long width. We have observed pulses with full widths at half-maximum between 100 and 1000 ns, which appears to scale at least roughly with the bubble size. A similar scaling with bubble size was observed in the water measurements, but the flash width there was 2-8 ns, about a hundred times smaller. The luminescence pulse in the liquid nitrogen is consistently observed only at pressures above $3-4$ bars [3]; at lower pressures $0.5-1$ bar it is more rare, occuring only for the largest bubbles where the pulse width is very long (1000 ns).

Fig. 2 shows photographs of the bubble, illustrating a) an expanding bubble pushing the ther- mal plume created by the focused YAG beam before it, b) shrinking just before the first collapse, and c) near the second collapse point, where considerable heating of the fluid is apparent from the convection tendrils in the region.

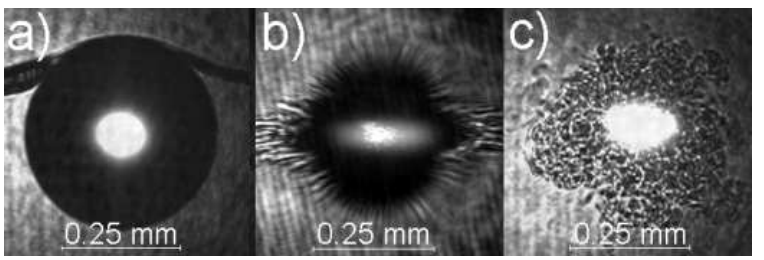

Fig. 2. Photographs of bubbles created in liquid $\mathrm{N}_{2}$ at 5.8 bar : a) bubble just after seeding, $\Delta \mathrm{t}=4 \mu$ s after the YAG pulse; b) bubble before the first collapse, $\Delta \mathrm{t}=45 \mu \mathrm{s}$; c) bubble region at $\Delta \mathrm{t}=90 \mu \mathrm{s}$, near the second collapse. (exposure time of all pictures: $1 \mu \mathrm{s}$ )

The results in liquid Ar at $84 \mathrm{~K}$ were completely similar to those shown here for liquid $\mathrm{N}_{2}$. The pulse widths were hundreds of nanoseconds, and it was again necessary to pressurize above a few bar to consistently see pulses at the bubble collapse point. However, preliminary measurements in liquid $\mathrm{He}$ and $\mathrm{O}_{2}$ do not show any luminescence pulses in those liquids for pressures up to $12 \mathrm{bar}$.

This work is supported by the U. S. National Science Foundation, DMR 97-31523. We thank M. Bernard and E. Varoquaux for initial development of this project. One of us (B.T.) thanks Deutsche Akad. der Naturforscher Leopoldina for support.

\section{References}

[1] D. F. Gaitan, L. A. Crum, R. A. Roy, C. C. Church, J. Acoust. Soc. Am. 91 (1992) 3166; B. P. Barber, R. Hiller, R. Lofstedt, S. Putterman, K. Weninger, Physics Reports 281 (1997) 65.

[2] P. Jarman, K. Taylor, J. Low Temp. Phys. 2 (1970) 389.

[3] P. I. Golubnichii, V. D. Goncharov, Kh. V. Protopopov, Sov. Phys. Acoust. 16 (1971) 323.

[4] O. Baghdassarian, H. Cho, E. Varoquaux, G. A. Williams, J. Low Temp. Phys. 110 (1998) 305.

[5] O. Baghdassarian, B. Tabbert, G. A. Williams, Phys. Rev. Lett. 83 (1999) 2437.

[6] C. D. Ohl, O. Lindau, W. Lauterborn, Phys. Rev. Lett. 80 (1998) 393. 